\title{
Types of Linkage of Quadratic Pfister Forms
}

\author{
Adam Chapman \\ Department of Computer Science, Tel-Hai Academic College, Upper Galilee, 12208 Israel \\ Andrew Dolphin \\ Departement Wiskunde-Informatica, Universiteit Antwerpen, Belgium
}

\begin{abstract}
Given a field $F$ of positive characteristic $p, \theta \in H_{p}^{n-1}(F)$ and $\beta, \gamma \in F^{\times}$, we prove that if the symbols $\theta \wedge \frac{d \beta}{\beta}$ and $\theta \wedge \frac{d \gamma}{\gamma}$ in $H_{p}^{n}(F)$ share the same factors in $H_{p}^{1}(F)$ then the symbol $\theta \wedge \frac{d \beta}{\beta} \wedge \frac{d \gamma}{\gamma}$ in $H_{p}^{n+1}(F)$ is trivial. We conclude that when $p=2$, every two totally separably $(n-1)$-linked $n$-fold quadratic Pfister forms are inseparably $(n-1)$ linked. We also describe how to construct non-isomorphic $n$-fold Pfister forms which are totally separably (or inseparably) $(n-1)$-linked, i.e. share all common $(n-1)$-fold quadratic (or bilinear) Pfister factors.
\end{abstract}

Keywords: Kato-Milne Cohomology, Fields of Positive Characteristic, Quadratic Forms, Pfister Forms, Quaternion Algebras, Linkage

2010 MSC: 11E81 (primary); 11E04, 16K20, 19D45 (secondary)

\section{Introduction}

Linkage of Pfister forms is a classical topic in quadratic form theory. We say that two $n$-fold Pfister forms over a field $F$ are separably (inseparably, resp.) $m$-linked if there exists an $m$-fold quadratic (bilinear) Pfister form which is a common factor of both forms. When $\operatorname{char}(F) \neq 2$, there is no difference between quadratic and bilinear factors, so the terms coincide, and we simply say $m$-linked.

We say that two quadratic $n$-fold Pfister forms are totally separably (inseparably) $m$-linked if every quadratic (bilinear) $m$-fold Pfister factor of one of them is also a factor of the other. The following facts were proven in [4]:

- Two $n$-fold quadratic Pfister forms can be totally separably 1-linked, inseparably 1-linked, or even both, without being isometric. (The special case of quaternion algebras over fields of characteristic not 2 was covered in [9].)

- Total separable 1-linkage and total inseparable 1-linkage of $n$-fold quadraticPfister forms are independent properties, i.e. do not imply each other.

Email addresses: adam1chapman@yahoo.com (Adam Chapman), Andrew.Dolphin@uantwerpen . be (Andrew Dolphin) 
Clearly total separable (or inseparable) $(n-1)$-linkage implies (nontotal) separable (inseparable, resp.) $(n-1)$-linkage. It is known that inseparable $(n-1)$-linkage of quadratic $n$-fold Pfister forms implies separable $(n-1)$-linkage, but the converse is in general not true (see [12], [8], [5], [6], [3] and [1] for references).

Question 1.1. Does total separable $(n-1)$-linkage of $n$-fold quadratic Pfister forms imply (nontotal) inseparable $(n-1)$-linkage?

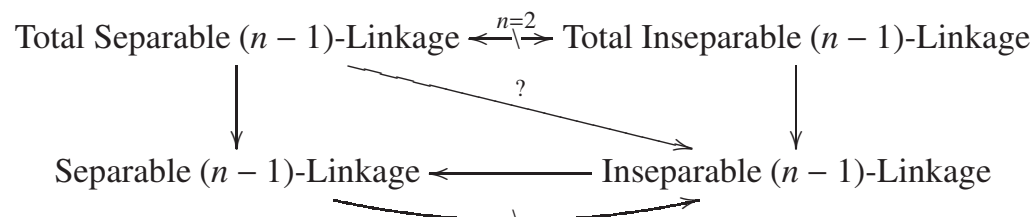

We answer this question in the positive in Section 3 . We conclude it from deeper results on linkage of symbols in the Kato-Milne cohomology groups.

There are several other natural questions that arise in this setting:

Question 1.2. Do there exist totally separably (inseparably) m-linked quadratic n-fold Pfister forms which are not isometric for a given $m \in\{1, \ldots, n-1\}$ ?

Question 1.3. Over fields of characteristic 2, are total separable m-linkage and total inseparable m-linkage independent properties?

Question 1.4. Given $1 \leqslant \ell<m \leqslant n-1$, are there totally separably (or inseparably) $\ell$-linked $n$-fold quadratic Pfister forms which are not totally separably (inseparably, resp.) m-linked?

We answer Question 1.2 in full generality (in the positive), and Question 1.4 in the case of fields of characteristic not 2 and $m=n-1$ (see Section 4). Question 1.3 was answered in the negative in [4] for $m=1$, but it remains open for arbitrary $m$.

\section{Preliminaries}

\subsection{Quadratic Forms}

For general reference on symmetric bilinear forms and quadratic forms see [7]. Throughout, let $F$ be a field and $V$ an $F$-vector space. A quadratic form over $F$ is a $\operatorname{map} \varphi: V \rightarrow F$ such that $\varphi(a v)=a^{2} \varphi(v)$ for all $a \in F$ and $v \in V$ and the map defined by $B_{\varphi}(v, w)=\varphi(v+w)-\varphi(v)-\varphi(w)$ for all $v, w \in V$ is a bilinear form on $V$. The bilinear form $B_{\varphi}$ is called the polar form of $\varphi$ and is clearly symmetric. Two quadratic forms $\varphi: V \rightarrow F$ and $\psi: W \rightarrow F$ are isometric if there exists an isomorphism $M: V \rightarrow W$ such that $\varphi(v)=\psi(M v)$ for all $v \in V$. We are interested in the isometry classes of quadratic forms, so when we write $\varphi=\psi$ we actually mean that they are isometric.

We say that $\varphi$ is singular if $B_{\varphi}$ is degenerate, and that $\varphi$ is nonsingular if $B_{\varphi}$ is nondegenerate. If $F$ is of characteristic 2, every nonsingular form $\varphi$ is even dimensional and can be written as

$$
\varphi=\left[\alpha_{1}, \beta_{1}\right] \perp \cdots \perp\left[\alpha_{n}, \beta_{n}\right]
$$


for some $\alpha_{1}, \ldots, \beta_{n} \in F$, where $[\alpha, \beta]$ denotes the two-dimensional quadratic form $\psi(x, y)=\alpha x^{2}+x y+\beta y^{2}$ and $\perp$ denotes the orthogonal sum of quadratic forms. If the characteristic of $F$ is different from 2, symmetric bilinear forms and quadratic forms are equivalent objects, and we do not distinguish between them in this case. The unique nonsingular two-dimensional isotropic quadratic form is $\mathbb{H}=[0,0]$, called the hyperbolic plane. A hyperbolic form is an orthogonal sum of hyperbolic planes. Any quadratic form $\varphi$ over $F$ decomposes into an orthogonal sum of a uniquely determined anisotropic quadratic form and a number of hyperbolic planes. The number of hyperbolic planes appearing in this decomposition is called the Witt index and denoted $i_{W}(\varphi)$.

We denote by $\left\langle\alpha_{1}, \ldots, \alpha_{n}\right\rangle$ the diagonal bilinear form given by $(x, y) \mapsto \sum_{i=1}^{n} \alpha_{i} x_{i} y_{i}$. A bilinear $n$-fold Pfister form over $F$ is a symmetric bilinear form isometric to a bilinear form

$$
\left\langle 1, \alpha_{1}\right\rangle \otimes\left\langle 1, \alpha_{2}\right\rangle \otimes \cdots \otimes\left\langle 1, \alpha_{n}\right\rangle
$$

for some $\alpha_{1}, \alpha_{2}, \ldots, \alpha_{n} \in F^{\times}$. We denote such a form by $\left\langle\left\langle\alpha_{1}, \alpha_{2}, \ldots, \alpha_{n}\right\rangle\right\rangle$. By convention, the bilinear 0 -fold Pfister form is $\langle 1\rangle$. The 2-fold Pfister forms generate the fundamental ideal $I F$ in the Witt ring of nondegenerate symmetric bilinear forms $W F$. Powers of $I F$ are denoted by $I^{n} F$, and are generated by $n$-fold Pfister forms respectively.

Let $B: V \times V \rightarrow F$ be a symmetric bilinear form over $F$ and $\varphi: W \rightarrow F$ be a quadratic form over $F$. We may define a quadratic form $B \otimes \varphi: V \otimes_{F} W \rightarrow F$ determined by the rule that $(B \otimes \varphi)(v \otimes w)=B(v, v) \cdot \varphi(w)$ for all $w \in W, v \in V$. We call this quadratic form the tensor product of $B$ and $\varphi$. A quadratic $n$-fold Pfister form over $F$ is a tensor product of a bilinear $(n-1)$-fold Pfister form $\left.\left\langle\alpha_{1}, \alpha_{2}, \ldots, \alpha_{n-1}\right\rangle\right\rangle$ and a two-dimensional quadratic form $[1, \beta]$ for some $\beta \in F$. We denote such a form by $\left\langle\left\langle\alpha_{1}, \ldots, \alpha_{n-1}, \beta \rrbracket\right.\right.$. Quadratic $n$-fold Pfister forms are isotropic if and only if they are hyperbolic (see [7, (9.10)]). The 2-fold quadratic Pfister forms generate the fundamental ideal, denoted $I_{q} F$ or $I_{q}^{1} F$, of the Witt group of nonsingular quadratic forms. Let $I_{q}^{n} F$ denote the subgroup generated by scalar multiples of quadratic $n$-fold Pfister forms.

Given a symmetric bilinear form $B$, we denote by $Q(B)$ the quadratic form given by the map $v \mapsto B(v, v)$.

Let $\pi$ be an $n$-fold quadratic Pfister form over $F$. For $m \in\{1, \ldots, n\}$, we say an $m$-fold quadratic (resp. bilinear) Pfister form $\psi$ (resp. $B$ ) is a factor of $\pi$ if there exists an $(n-m)$-fold bilinear (reps. quadratic) Pfister form $B^{\prime}$ (resp. $\psi^{\prime}$ ) such that $\pi=B^{\prime} \otimes \psi$ (resp. $\pi=B \otimes \psi^{\prime}$ ).

Let $\omega$ be an $n$-fold quadratic Pfister form over $F$. We say $\pi$ and $\omega$ are separably (resp. inseparably) $m$-linked if there exists an $m$-fold quadratic (resp. bilinear) Pfister form $\psi$ such that $\psi$ is a factor of both $\pi$ and $\omega$. We say $\pi$ and $\omega$ are totally separably (resp. inseparably) $m$-linked if every quadratic (resp. bilinear) $m$-fold Pfister form is a factor of $\pi$ if and only if it is a factor of $\omega$. This terminology comes from the fact that in characteristic 2, the function fields of quadratic (resp. bilinear) Pfister forms are separable (resp. inseparable) extensions of the ground field.

\subsection{Kato-Milne Cohomology}

In this section, assume $F$ is a field of characteristic $p>0$. For $n>0$, the KatoMilne Cohomology group $H_{p}^{n+1}(F)$ is defined to be the cokernel of the Artin-Schreier 
map

$$
\begin{gathered}
\wp: \Omega_{F}^{n} \rightarrow \Omega_{F}^{n} / d \Omega_{F}^{n-1} \\
\alpha \frac{d \beta_{1}}{\beta_{1}} \wedge \cdots \wedge \frac{d \beta_{n}}{\beta_{n}} \mapsto\left(\alpha^{p}-\alpha\right) \frac{d \beta_{1}}{\beta_{1}} \wedge \cdots \wedge \frac{d \beta_{n}}{\beta_{n}} .
\end{gathered}
$$

We also fix $H_{p}^{1}(F)$ to be $F / \wp(F)$. The group $v_{F}(n)$ is defined to be the kernel of this map. By [2], $v_{F}(n) \cong K_{n} F / p K_{n} F$, with the isomorphism given by

$$
\frac{d \beta_{1}}{\beta_{1}} \wedge \cdots \wedge \frac{d \beta_{n}}{\beta_{n}} \mapsto\left\{\beta_{1}, \ldots, \beta_{n}\right\} .
$$

It is known that $H_{p}^{2}(F) \cong{ }_{p} B r(F)$ and for $p=2, H_{2}^{n}(F) \cong I_{q}^{n} F / I_{q}^{n+1} F$. The first isomorphism is given by the map

$$
\alpha \frac{d \beta}{\beta} \mapsto[\alpha, \beta)_{p, F}
$$

where $[\alpha, \beta)_{p, F}$ stands for the symbol $p$-algebra

$$
F\left\langle x, y: x^{p}-x=\alpha, y^{p}=\beta, y x y^{-1}=x+1\right\rangle .
$$

The second isomorphism is given by the map

$$
\alpha \frac{d \beta_{1}}{\beta_{1}} \wedge \cdots \wedge \frac{d \beta_{n}}{\beta_{n}} \mapsto\left\langle\beta_{1}, \ldots, \beta_{n}, \alpha \rrbracket .\right.
$$

We call the logarithmic differentials $\alpha \frac{d \beta_{1}}{\beta_{1}} \wedge \cdots \wedge \frac{d \beta_{n-1}}{\beta_{n-1}}$ in $H_{p}^{n}(F)$ and $\frac{d \gamma_{1}}{\gamma_{1}} \wedge \cdots \wedge \frac{d \gamma_{m}}{\gamma_{m}}$ in $\nu_{F}(m)$ "symbols". There is a natural map

$$
H_{p}^{n}(F) \times v_{F}(m) \rightarrow H_{p}^{m+n}(F)
$$

defined by the wedge product

$$
\left(\alpha \frac{d \beta_{1}}{\beta_{1}} \wedge \cdots \wedge \frac{d \beta_{n-1}}{\beta_{n-1}}, \frac{d \gamma_{1}}{\gamma_{1}} \wedge \cdots \wedge \frac{d \gamma_{m}}{\gamma_{m}}\right) \mapsto \alpha \frac{d \beta_{1}}{\beta_{1}} \wedge \cdots \wedge \frac{d \beta_{n-1}}{\beta_{n-1}} \wedge \frac{d \gamma_{1}}{\gamma_{1}} \wedge \cdots \wedge \frac{d \gamma_{m}}{\gamma_{m}} .
$$

We define the linkage of symbols in an analogous manner to the linkage of Pfister forms. If a symbol $\omega$ in $H_{p}^{m+n}(F)$ is a wedge product $\theta \wedge \psi$ where $\theta$ is a symbol in $H_{p}^{n}(F)$ and $\psi$ is a symbol in $v_{F}(m)$, then $\theta$ and $\psi$ are called factors of $\omega$. We say that two symbols $\pi$ and $\omega$ are separably $k$-linked if they have a common factor in $H_{p}^{k}(F)$, and inseparably $k$-linked if they have a common factor in $v_{F}(k)$. We say that two symbols $\pi$ and $\omega$ are totally separably $k$-linked if they share all factors in $H_{p}^{k}(F)$, and inseparably $k$-linked if they share all factor in $v_{F}(k)$.

\section{Separably $(n-1)$-linked Symbols in $H_{p}^{n}(F)$}

In this section, assume $F$ is a field of characteristic $p>0$. One of the main goals is to show that total separable $(n-1)$-linkage implies inseparable $(n-1)$-linkage for quadratic $n$-fold Pfister forms when $p=2$. 
Lemma 3.1. For $\alpha \in F$ and $\beta \in F^{\times}$, let

$$
t=\alpha+\frac{(\alpha-\beta)}{\gamma} .
$$

The symbol p-algebra $[\alpha, \gamma)_{p, F}$ contains the étale extension $F\left[x: x^{p}-x=t^{p} \gamma+\beta\right]$ of $F$.

Proof. Let $i$ and $j$ be a pair of generators of $[\alpha, \gamma)_{p, F}$ with $i^{p}-i=\alpha, j^{p}=\gamma$ and $j i j^{-1}=i+1$. Take $x=i+t j+i j$ in $[\alpha, \gamma)_{p, F}$. Then $x^{p}-x$ is equal to

$$
\gamma \alpha^{p}+\gamma^{1-p} \alpha^{p}-\gamma^{1-p} \beta^{p}+\beta=t^{p} \gamma+\beta
$$

by [3, Lemma 3.1]. Hence the subalgebra $F[x]$ of $[\alpha, \gamma)_{p, F}$ is as required.

Proposition 3.2. Consider two separably $(n-1)$-linked symbols

$$
\pi=\alpha \frac{d \delta_{1}}{\delta_{1}} \wedge \cdots \wedge \frac{d \delta_{n-2}}{\delta_{n-2}} \wedge \frac{d \beta}{\beta} \quad \text { and } \quad \omega=\alpha \frac{d \delta_{1}}{\delta_{1}} \wedge \cdots \wedge \frac{d \delta_{n-2}}{\delta_{n-2}} \wedge \frac{d \gamma}{\gamma}
$$

in $H_{p}^{n}(F)$ and let $t=\alpha+\frac{(\alpha-\beta)}{\gamma}$. If $t^{p} \gamma+\beta$ is a factor in $H_{p}^{1}(F)$ of $\pi$, then the class of $\alpha \frac{d \delta_{1}}{\delta_{1}} \wedge \cdots \wedge \frac{d \delta_{n-2}}{\delta_{n-2}} \wedge \frac{d \beta}{\beta} \wedge \frac{d \gamma}{\gamma}$ in $H_{p}^{n+1}(F)$ is trivial.

Proof. Note first that if $t^{p} \gamma+\beta=0$ then $d \gamma \wedge d \beta=0$ and the result holds. Assume otherwise. The class of $t^{p} \gamma+\beta$ in $H_{p}^{1}(F)$ is a factor of $\omega$ by Lemma 3.1, so it is a common factor of $\pi$ and $\omega$. We have

$$
\alpha \frac{d \beta}{\beta} \wedge \frac{d \gamma}{\gamma}=\alpha \frac{d\left(\beta \gamma^{-1}\right)}{\beta \gamma^{-1}} \wedge \frac{d\left(t^{p} \gamma+\beta\right)}{t^{p} \gamma+\beta}
$$

(see [6, Lemma 5.1, (e)]). Now,

$$
\begin{gathered}
\alpha \frac{d \delta_{1}}{\delta_{1}} \wedge \cdots \wedge \frac{d \delta_{n-2}}{\delta_{n-2}} \wedge \frac{d \beta}{\beta} \wedge \frac{d \gamma}{\gamma}= \\
\alpha \frac{d \delta_{1}}{\delta_{1}} \wedge \cdots \wedge \frac{d \delta_{n-2}}{\delta_{n-2}} \wedge \frac{d \beta \gamma^{-1}}{\beta \gamma^{-1}} \wedge \frac{d\left(t^{p} \gamma+\beta\right)}{t^{p} \gamma+\beta}= \\
\alpha \frac{d \delta_{1}}{\delta_{1}} \wedge \cdots \wedge \frac{d \delta_{n-2}}{\delta_{n-2}} \wedge \frac{d \beta}{\beta} \wedge \frac{d\left(t^{p} \gamma+\beta\right)}{t^{p} \gamma+\beta}-\alpha \frac{d \delta_{1}}{\delta_{1}} \wedge \cdots \wedge \frac{d \delta_{n-2}}{\delta_{n-2}} \wedge \frac{d \gamma}{\gamma} \wedge \frac{d\left(t^{p} \gamma+\beta\right)}{t^{p} \gamma+\beta} .
\end{gathered}
$$

Since $t^{p} \gamma+\beta$ is a factor in $H_{p}^{1}(F)$ of $\alpha \frac{d \delta_{1}}{\delta_{1}} \wedge \cdots \wedge \frac{d \delta_{n-2}}{\delta_{n-2}} \wedge \frac{d \beta}{\beta}$, we have

$$
\alpha \frac{d \delta_{1}}{\delta_{1}} \wedge \cdots \wedge \frac{d \delta_{n-2}}{\delta_{n-2}} \wedge \frac{d \beta}{\beta} \wedge \frac{d\left(t^{p} \gamma+\beta\right)}{t^{p} \gamma+\beta}=\left(t^{p} \gamma+\beta\right) \tau \wedge \frac{d\left(t^{p} \gamma+\beta\right)}{t^{p} \gamma+\beta}
$$

for some $\tau \in v_{F}(n-1)$. As $\left(t^{p} \gamma+\beta\right) \frac{d\left(t^{p} \gamma+\beta\right)}{t^{p} \gamma+\beta}=d\left(t^{p} \gamma+\beta\right)$, it is trivial in $H_{p}^{2}(F)$. Hence $\alpha \frac{d \delta_{1}}{\delta_{1}} \wedge \cdots \wedge \frac{d \delta_{n-2}}{\delta_{n-2}} \wedge \frac{d \beta}{\beta} \wedge \frac{d\left(t^{p} \gamma+\beta\right)}{t^{p} \gamma+\beta}=0$. Similarly, since $t^{p} \gamma+\beta$ is a factor in $H_{p}^{1}(F)$ of $\alpha \frac{d \gamma}{\gamma}$, we have $\alpha \frac{d \delta_{1}}{\delta_{1}} \wedge \cdots \wedge \frac{d \delta_{n-2}}{\delta_{n-2}} \wedge \frac{d \gamma}{\gamma} \wedge \frac{d\left(t^{p} \gamma+\beta\right)}{t^{\gamma} \gamma+\beta}=0$. Therefore $\alpha \frac{d \delta_{1}}{\delta_{1}} \wedge \cdots \wedge \frac{d \delta_{n-2}}{\delta_{n-2}} \wedge \frac{d \beta}{\beta} \wedge \frac{d \gamma}{\gamma}=0$ in $H_{p}^{n+1}(F)$ as required. 
Corollary 3.3. Let

$$
\pi=\alpha \frac{d \delta_{1}}{\delta_{1}} \wedge \cdots \wedge \frac{d \delta_{n-2}}{\delta_{n-2}} \wedge \frac{d \beta}{\beta} \quad \text { and } \quad \omega=\alpha \frac{d \delta_{1}}{\delta_{1}} \wedge \cdots \wedge \frac{d \delta_{n-2}}{\delta_{n-2}} \wedge \frac{d \gamma}{\gamma}
$$

be two separably $(n-1)$-linked symbols in $H_{p}^{n}(F)$. If $\pi$ and $\omega$ are totally separably 1-linked then the class of

$$
\alpha \frac{d \delta_{1}}{\delta_{1}} \wedge \cdots \wedge \frac{d \delta_{n-2}}{\delta_{n-2}} \wedge \frac{d \beta}{\beta} \wedge \frac{d \gamma}{\gamma}
$$

in $H_{p}^{n+1}(F)$ is trivial.

When $p=2$, by the identification of the symbols with quadratic $n$-fold Pfister forms, we obtain the following results:

Proposition 3.4. Assume $p=2$. Let

$$
\pi=\left\langle\left\langle\beta, \delta_{n-2}, \ldots, \delta_{1}, \alpha \rrbracket \quad \text { and } \quad \omega=\left\langle\gamma \gamma, \delta_{n-2}, \ldots, \delta_{1}, \alpha \rrbracket\right.\right.\right.
$$

be two separably $(n-1)$-linked $n$-fold quadratic Pfister forms over $F$ and let $t=\alpha+$ $\frac{(\alpha-\beta)}{\gamma}$. If the 1-fold Pfister form $\left\langle t^{p} \gamma+\beta \rrbracket\right.$ is a factor of $\pi$, then $\left\langle\beta \beta, \gamma, \delta_{n-2}, \ldots, \delta_{1}, \alpha \rrbracket\right.$ is trivial. In particular, $\pi$ and $\omega$ are inseparably $(n-1)$-linked.

Proof. By [5], the $(n+1)$-fold Pfister form $\left\langle\beta, \gamma, \delta_{n-1}, \ldots, \delta_{2}, \alpha \rrbracket\right.$ is trivial if and only if $\pi$ and $\omega$ are inseparably $(n-1)$-linked.

Corollary 3.5. Assume $p=2$. If a pair of separably $(n-1)$-linked $n$-fold quadratic Pfister forms over $F$ are totally separably 1-linked then they are also inseparably ( $n-$ 1)-linked. In particular, if a pair of n-fold quadratic Pfister forms over $F$ are totally separably $(n-1)$-linked then they are inseparably $(n-1)$-linked.

Remark 3.6. A similar result to Corollary 3.3 holds more straight-forwardly for Milnor $K$-groups. Let $p$ be a prime integer, $n$ a positive integer and $F$ an arbitrary field. If $p=2$ then further assume that $\sqrt{-1} \in F$. Then the following is trivial:

- If $\{\alpha\} \cup \theta \in K_{n} F / p K_{n} F$ has $\{\beta\}$ as a factor in $K_{1} F / p K_{1} F$ then $\{\alpha, \beta\} \cup \theta=0$ in $K_{n+1} F / p K_{n+1} F$.

- Therefore, if $\{\alpha\} \cup \theta$ and $\{\beta\} \cup \theta$ in $K_{n} F / p K_{n} F$ are totally 1-linked then $\{\alpha, \beta\} \cup \theta=$ 0 in $K_{n+1} F / p K_{n+1} F$.

Remark 3.7. The result analogous to Corollary 3.3 for inseparable linkage is also straight-forward. For fields $F$ of characteristic $p>0$ and positive integer $n$,

- If $\omega \wedge \frac{d \beta}{\beta} \in H_{p}^{n} F$ has $\frac{d \gamma}{\gamma}$ in $v_{F}(1)$ as a factor then $\omega \wedge \frac{d \beta}{\beta} \wedge \frac{d \gamma}{\gamma}=0$ in $H_{p}^{n+1}(F)$.

- Therefore, if $\omega \wedge \frac{d \beta}{\beta}$ and $\omega \wedge \frac{d \gamma}{\gamma}$ in $H_{p}^{n} F$ are totally inseparably 1-linked then $\omega \wedge \frac{d \beta}{\beta} \wedge \frac{d \gamma}{\gamma}=0$ in $H_{p}^{n+1}(F)$. 


\section{Totally Linked Quadratic Pfister Forms}

In [4] we considered whether total 1-linkage of Pfister forms implied isometry. In general it does not. In this section, we consider whether total $m$-linkage implies isometry.

Lemma 4.1. Let $n$ be an integer $\geqslant 2$ and $m \in\{1, \ldots, n-1\}$. Let $\varphi$ be an $n$-fold quadratic Pfister form, $\pi$ an $m$-fold quadratic Pfister form and $\theta$ an ( $m-1)$-fold quadratic Pfister form. Assume $\theta$ is a common factor of $\varphi$ and $\pi$. Then $i_{W}(\varphi \perp-\pi)=2^{m}$ if and only if $\pi$ is a factor of $\varphi$. Otherwise, $i_{W}(\varphi \perp-\pi)=2^{m-1}$.

Proof. By [7, Corollary 24.3], $i_{W}(\varphi \perp-\pi)$ must be a power of 2. Since $\theta$ is a factor of $\varphi, i_{W}(\varphi \perp-\pi) \geqslant 2^{m-1}$. Therefore, the only other possible value is $2^{m}$, in which case $\pi$ is a subform of $\varphi$ and therefore a factor of $\varphi$.

Lemma 4.2. Let $n$ be an integer $\geqslant 2$ and $m \in\{1, \ldots, n-1\}$. Let $\varphi$ and $\psi$ be two nonhyperbolic, separably $(n-1)$-linked and totally separably m-linked $n$-fold quadratic Pfister forms over $F$. Let $\pi$ be an $(m+1)$-fold quadratic Pfister form such that $\pi$ is a factor of $\varphi$ but not $\psi$. Then there exists a field extension $L$ such that $\pi_{L}$ is a factor of $\psi_{L}$ and $\varphi_{L}$ and $\psi_{L}$ are neither isometric nor hyperbolic.

Proof. Since $\varphi$ and $\psi$ are separably ( $n-1)$-linked, the form $\varphi \perp \psi$ is congruent mod $I_{q}^{n+1} F$ to some anisotropic $n$-fold Pfister form $\phi$. Let $\pi_{0}$ be an $m$-fold quadratic Pfister factor of $\pi$. Since $\varphi$ and $\psi$ are totally separably $m$-linked, $\pi_{0}$ is a common factor of both forms.

By Lemma 4.1, $\psi \perp-\pi$ is Witt equivalent to some anisotropic $2^{n}$-dimensional form $\theta$. Write $L=F(\theta)$ for the function field of $\theta$ over $F$. If one of the forms $\varphi_{L}, \psi_{L}$ and $\phi_{L}$ were hyperbolic, then $\theta$ would be similar to a subform of the form by $[7$, Corollary 22.5]. However, since the forms are of the same dimension, this would imply that $\theta$ is similar to an $n$-fold Pfister form. This is impossible because the $m$ th cohomological invariant of $\theta$ is nontrivial. It follows that $\psi_{L}$ and $\varphi_{L}$ are not isometric as $\phi_{L}$ is not hyperbolic.

Theorem 4.3. Let $n$ be an integer $\geqslant 2$ and $m \in\{1, \ldots, n-1\}$. Let $\varphi$ and $\psi$ be two non-hyperbolic, separably $(n-1)$-linked and totally separably m-linked quadratic $n$ fold Pfister forms over $F$. Then there exists a field extension $K$ of $F$ such that $\varphi_{K}$ and $\psi_{K}$ are totally separably $(m+1)$-linked but not isometric nor hyperbolic.

Proof. Let $S$ be the set of $(m+1)$-fold quadratic Pfister forms $\pi$ over $F$ such that $\pi$ is a factor of $\varphi$ but not of $\psi$. Then as in Lemma 4.2 for each $\pi \in S$, there exists a $2^{n}$-dimensional quadratic form $\theta$ such that $\theta$ is Witt equivalent to $\pi \perp \psi$. Let $F_{0}$ be the compositum of the function fields of all such $\theta$.

Similarly, let $T$ be the set of $(m+1)$-fold quadratic Pfister forms $\pi^{\prime}$ over $F$ such that $\pi$ is a factor of $\psi$ but not of $\varphi$. Again, as in Lemma 4.2 for each $\pi^{\prime} \in T$, there exists a $2^{n}$-dimensional quadratic form $\theta^{\prime}$ such that $\theta^{\prime}$ is Witt equivalent to $\pi^{\prime} \perp \varphi$. Let $F_{0}^{\prime}$ be the field compositum of the function fields of all such $\theta^{\prime}$.

Let $K_{0}$ be the compositum of $F_{0}$ and $F_{0}^{\prime}$. Then by Lemma 4.2, $\varphi_{K_{0}}$ and $\psi_{K_{0}}$ are not isometric nor hyperbolic, and every $(m+1)$-fold Pfister form over $K_{0}$ defined over $F$ is 
a factor of $\varphi_{K_{0}}$ if and only if it is a factor of $\psi_{K_{0}}$. Using this construction inductively, we obtain the required field extension $K / F$.

Lemma 4.4. Assume $\operatorname{char}(F)=2$. Let $\theta$ be an anisotropic $n$-fold bilinear Pfister form over $F$ and let $\theta^{\prime}$ be an $(n-1)$-fold bilinear Pfister form factor of $\theta$. Let $\beta$ be an element represented by $\theta$ but not by $\theta^{\prime}$. Then $Q(\theta)=Q\left(\langle\beta \beta\rangle \otimes \theta^{\prime}\right)$.

Proof. This follows easily from [7, Proposition 10.4].

Lemma 4.5. Assume $\operatorname{char}(F)=2$. Let $n$ be an integer $\geqslant 2$ and $m \in\{1, \ldots, n-1\}$. Let $\varphi$ be an anisotropic $n$-fold quadratic Pfister form, $\pi$ an anisotropic $m$-fold bilinear Pfister form and $\theta$ an $(m-1)$-fold bilinear Pfister form. Assume $\theta$ is a common factor of $\varphi$ and $\pi$. Then $i_{W}(\varphi \perp Q(\pi))=2^{m}$ if and only if $\pi$ is a factor of $\varphi$. Otherwise, $i_{W}(\varphi \perp Q(\pi))=2^{m-1}$.

Proof. Since $\theta$ is a factor of $\varphi$, there exists a $2^{n-m}-1$ dimensional bilinear form $b$ and a quadratic 1-fold Pfister form $\rho$ such that

$$
\varphi=\theta \otimes(\langle 1\rangle \perp b) \otimes \rho .
$$

Set $\tau=\theta \otimes b \otimes \rho$ (note that $b \otimes \rho$ is the so-called pure part of the quadratic Pfister form $(\langle 1\rangle \perp b) \otimes \rho$, see [7, p.66]). Then we have that

$$
\varphi \perp Q(\theta)=2^{m-1} \otimes \mathbb{H} \perp \tau \perp Q(\theta) .
$$

Note that $\tau \perp Q(\theta)$ is anisotropic as $\varphi$ is aniostropic.

Suppose $\tau \perp Q(\pi)$ is isotropic. Since $\tau \perp Q(\theta)$ is anisotropic, there exists an element $\beta$ represented by $\tau$ and $Q(\pi)$ but not by $Q(\theta)$. As $\beta$ is represented by $Q(\pi)$ but not by $Q(\theta)$, it follows from Lemma 4.4 that $Q(\langle\beta\rangle\rangle \otimes \theta)=Q(\pi)$. As $\beta$ is represented by $Q(\tau)$ but not by $Q(\theta)$, it follows from [7, Proposition 15.7] that $\langle\langle\beta\rangle \otimes \theta$ is a factor of $\varphi$. In particular, $\varphi$ becomes isotropic over the function field of $\pi$. Hence $\pi$ is a factor of $\varphi$ by [11, (1.4)] and repeated use of [7, (15.6)].

Theorem 4.6. Assume $\operatorname{char}(F)=2$ and $n$ be an integer $\geqslant 2$ and $m \in\{1, \ldots, n-1\}$. Let $\varphi$ and $\psi$ be two non-hyperbolic, separably $(n-1)$-linked and totally inseparably $m$-linked $n$-fold quadratic Pfister forms over $F$. Then there exists a field extension $K$ of $F$ such that $\varphi_{K}$ and $\psi_{K}$ are totally inseparably $(m+1)$-linked but neither isometric nor hyperbolic.

Proof. The result follows from Lemma 4.5 in a similar way to Theorem 4.3 .

Using Theorems 4.3 and 4.6 one can construct examples of non-isometric pairs of $n$-fold quadratic Pfister forms which are totally separably (or inseparably, or both) $m$-linked for any $m \in\{1, \ldots, n-1\}$.

Example 4.7. Start with the field $F=\mathbb{F}_{2}\left(\left(x_{1}\right)\right) \ldots\left(\left(x_{n+1}\right)\right)$ of iterated Laurent series in $n+1$ indeterminates over $\mathbb{F}_{2}$. The forms

$$
\varphi=\left\langle\left\langle x_{1}, \ldots x_{n-1}, x_{1} \cdot \ldots \cdot x_{n} \rrbracket \quad \text { and } \quad \psi=\left\langle\left\langle x_{2}, \ldots x_{n}, x_{2} \cdot \ldots \cdot x_{n+1} \rrbracket\right.\right.\right.\right.
$$

over $F$ are $(n-1)$-linked but not totally 1-linked (see [5, Sections 9\&10]). By iterating Theorem 4.3 (or 4.6) $m$ times, we end up with a field $K$ over which $\varphi_{K}$ and $\psi_{K}$ are totally $m$-linked, but neither isometric nor hyperbolic. 
Question 4.8. When $\operatorname{char}(F)=p$, does there exist a similar process that extends two non-equal, nontrivial separably $(n-1)$ symbols in $H_{p}^{n}(F)$ to two non-equal, nontrivial totally separably (or inseparably) $(n-1)$-linked symbols?

Theorem 4.9. Assume char $(F) \neq 2$ and $n$ be an integer $\geqslant 3$ and $m \in\{1, \ldots, n-3\}$. Let $\varphi$ and $\psi$ be two non-hyperbolic (n-1)-linked and totally m-linked $n$-fold quadratic Pfister forms over $F$. Assume there exists an ( $n-1)$-fold quadratic Pfister form $\omega$ such that

(a) $\omega$ is a factor of $\varphi$,

(b) $\omega$ is not a factor of $\psi$,

(c) there exists an (n-2)-fold quadratic Pfister form that is a factor of both $\omega$ and $\psi$.

Then there exists a field extension $K$ of $F$ such that $\varphi_{K}$ and $\psi_{K}$ are totally $(m+1)$-linked but not totally $(n-1)$-linked nor hyperbolic.

Proof. This is essentially the same proof as in Theorem 4.3 Here we just need to note the following: The form $\psi \perp-\omega$ is Witt equivalent to some anisotropic $2^{n}$ dimensional form. The latter remains anisotropic under scalar extension to $L$ by [10, Theorem 5.4]. Therefore $\omega_{L}$ is not a subform of $\psi_{L}$, and that completes the proof.

Example 4.10. Start with the field $F=\mathbb{C}\left(\left(x_{1}\right)\right) \ldots\left(\left(x_{n+1}\right)\right)$ of iterated Laurent series in $n+1$ indeterminates over $\mathbb{C}$. The forms $\varphi=\left\langle\left\langle x_{1}, \ldots, x_{n}\right\rangle\right\rangle$ and $\psi=\left\langle\left\langle x_{2}, \ldots, x_{n+1}\right\rangle\right\rangle$ over $F$ are $(n-1)$-linked but not totally 1 -linked. By iterating Theorem $4.3 \mathrm{~m}$ times, we end up with a field $K$ over which $\varphi_{K}$ and $\psi_{K}$ are totally $m$-linked, but neither hyperbolic nor isometric. If $m \leqslant n-2$ then by Theorem 4.9, $\varphi_{K}$ and $\psi_{K}$ are also not totally $(n-1)$-linked.

\section{Acknowledgements}

The authors thank Jean-Pierre Tignol for his comments on the manuscript. We also thank the annoymous referee for a careful reading of the manuscript and helpful suggestions for its improvement.

\section{References}

[1] C. Beli, P. Gille, and T.-Y. Lee. Examples of algebraic groups of type $G_{2}$ having the same maximal tori. Proc. Steklov Inst. Math., 292(1):10-19, 2016.

[2] S. Bloch and K. Kato. p-adic étale cohomology. Inst. Hautes Études Sci. Publ. Math., 63:107-152, 1986.

[3] A. Chapman. Common subfields of p-algebras of prime degree. Bull. Belg. Math. Soc. Simon Stevin, 22(4):683-686, 2015.

[4] A. Chapman, A. Dolphin, and A. Laghribi. Total linkage of quaternion algebras and Pfister forms in characteristic two. J. Pure Appl. Algebra, 220(11):36763691, 2016. 
[5] A. Chapman, S. Gilat, and U. Vishne. Linkage of quadratic Pfister forms. Comm. Algebra, 45(12):5212-5226, 2017.

[6] A. Chapman and K. McKinnie. Kato-Milne cohomology and polynomial forms. J. Pure Appl. Algebra, 222(11):3547-3559, 2018.

[7] R. Elman, N. Karpenko, and A. Merkurjev. The algebraic and geometric theory of quadratic forms, volume 56 of American Mathematical Society Colloquium Publications. American Mathematical Society, Providence, RI, 2008.

[8] F. Faivre. Liaison des formes de Pfister et corps de fonctions de quadriques en caractéristique 2. 2006. Thesis (Ph.D.)-Université de Franche-Comté.

[9] S. Garibaldi and D. J. Saltman. Quaternion algebras with the same subfields. In Quadratic forms, linear algebraic groups, and cohomology, volume 18 of Dev. Math., pages 225-238. Springer, New York, 2010.

[10] D. W. Hoffmann. Twisted Pfister forms. Doc. Math., 1:No. 03, 67-102, 1996.

[11] A. Laghribi. Witt kernels of function field extensions in characteristic 2. J.Pure Appl. Algebra, 199, 167-182, 2005.

[12] T. Y. Lam. On the linkage of quaternion algebras. Bull. Belg. Math. Soc. Simon Stevin, 9(3):415-418, 2002. 